\title{
The relationship between synapsis and chiasma distribution in grasshopper bivalents heterozygous for supernumerary segments
}

\author{
J. L. SANTOS, A. DEL CERRO, A. FERNÁNDEZ \& M. DÍEZ \\ Departamento Genética, Facultad de Biologia, Universidad Complutense, 28040 Madrid, Spain
}

\begin{abstract}
The relationship between chiasma distributions and pairing behaviour of grasshopper bivalent heterozygous for supernumerary segments has been analysed. The extra segments were located at the telomeric end of the long arm in chromosome S8 of Chorthippus jacobsi and at interstitial position in chromosomes S8 and M7 of Euchorthippus pulvinatus. In all cases these heteromorphic bivalents exhibited fully paired synaptonemal complexes with equalized axial lengths either at late zygotene or early pachytene, that is, heterosynapsis in regions near the extra segments is achieved at these meiotic stages. It is proposed that the absence of chiasmata in those regions in which nonhomologous pairing took place, would lead to chiasma redistributions observed in these bivalents when compared with those observed in bivalents in which extra segments are lacking.
\end{abstract}

Keywords: chiasma distribution, meiosis, supernumerary segments, surface spreading, synapsis.

\section{Introduction}

Populations of grasshoppers frequently show differential polymorphism with respect to the presence and frequency of supernumerary segments on one or more members of the standard complement. This extra material is in most cases of heterochromatic nature and is located on small chromosomes in proximal, interstitial or distal regions.

The main effect of these segments is at the endophenotypic level, mainly on the recombination process. In fact, they can increase the mean cell chiasma frequency of the male carriers when compared to noncarriers (see for instance Hewitt \& John, 1968; Riva et al., 1984) and produce changes in chiasma distribution of the bivalents bearing them in such a way that regions furthest from the segment are preferred for chiasma formation (Miklos \& Nankivell, 1976; John \& King, 1985; Navas-Castillo et al., 1985).

It has recently been proposed that the redistribution of chiasmata in bivalents with polymorphic ends could be produced by the misalignment of homologous chromosomes where they are attached to the nuclear envelope (Moens et al., 1989). In this paper we provide experimental data on chiasma distributions and pairing behaviour of bivalents heterozygous for supernumerary segments and bivalents without such segments in two species of grasshopper, Chorthippus jacobsi and Euchorthippus pulvinatus.

\section{Materials and methods}

Testes of three males heterozygous for a supernumerary segment in the S8 chromosome (BS) and of five males without the segment (basic homozygotes, BB) of Chorthippus jacobsi and testes of one male heterozygous for a segment in the M7 chromosome (BS), of two males heterozygous for a segment in the S8 chromosome and of three basic homozygotes of Euchorthippus pulvinatus were used in this study. They were selected from last instar nymphs collected at the Campus of Complutense University (1990) and Valdemoro (Madrid, 1990) respectively.

Testes were divided into two parts, one fixed in ethanol-glacial acetic acid $(3: 1)$ and stored at $4^{\circ} \mathrm{C}$. Preparations were made in 45 per cent acetic acid and, after removing the coverslips, slides were air dried and stained by the C-banding procedure described by Santos \& Giráldez (1978). The other part was subjected to a surface spreading procedure according to the method of Croft \& Jones (1986), with some modifications. Briefly: testes, without the fat body, were homogenized in Ham's F12 medium. Two drops of detergent solution $(0.03$ per cent Joy in distilled water, $\mathrm{pH}=7.5$ ) were added to a drop of homogenized testis suspension on a cavity slide, left for $5 \mathrm{~min}$ and washed onto plastic-coated slides with seven drops of paraformaldehyde fixative $(\mathrm{pH}=8.9)$. The preparations were then dried overnight on a warm plate at $30-35^{\circ} \mathrm{C}$, 
rinsed and air dried. For silver impregnation, a few drops of 50 per cent $\mathrm{AgNO}_{3}$ solution were placed on the slide and covered with a patch of nylon cloth. Nuclei were examined using a Zeiss EM 902 and photographed on Kodak 4489 film.

The position of the single chiasma in M7 and S8 bivalents was classified by eye as proximal, interstitial or distal with respect to the centromere. Data from different individuals with the same C-banding pattern for the chromosome analysed (M7 or S8) have been pooled because they showed similar patterns of chiasma distribution.

\section{Results}

\section{Chorthippus jacobsi}

As in other gomphocerine grasshoppers, this species has 17 chromosomes $(16+\mathrm{X})$ in the male and 18 $(16+\mathrm{XX})$ in the female. Three pairs of autosomes are long and submetacentric and the remaining five pairs of autosomes and the $\mathrm{X}$-chromosome are of medium to small size and acrocentric. Giemsa C-banding revealed centromeric heterochromatin in all chromosomes and telomeric heterochromatin in M5, M6, M7 and S8 chromosomes. Quantitative heterochromatin variation was observed in the M7 chromosome. In M5, M6 and S8 chromosomes telomeric heterochromatin was also variable in size and could even be absent. Figure 1 shows a metaphase I cell in which a S8 heteromorphic bivalent is observed.

One chiasma is invariably formed in S8 bivalents independently of their heterochromatin constitution. The chiasma distributions in two classes of S8 bivalents, homozygotes without the segment (BB) and heterozygotes (BS) are shown in Fig. 2. In S8 BB bivalents, chiasmata were preferentially located at interstitial and distal positions whereas in BS bivalents an

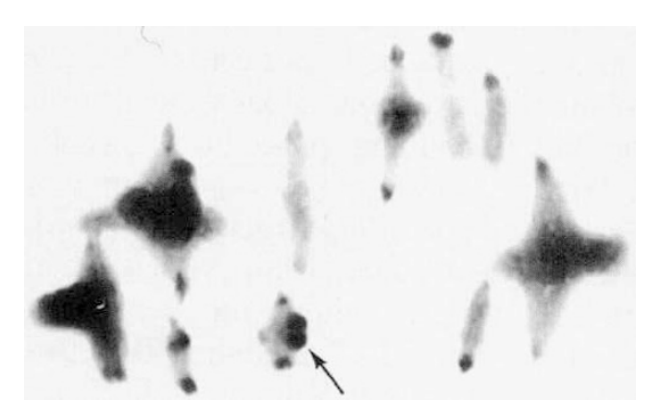

Fig. 1 C-banded metaphase I cell of a Chorthippus jacobsi male heterozygous for a supernumerary segment in the S 8 bivalent (arrowed). increase in proximal and interstitial chiasmata and a parallel decrease in distal ones was observed.

Analysis of zygotene surface spread nuclei in this species, as in other grasshoppers, is complicated due to difficulties in tracing axial and lateral elements because they are very thin and in many cases they can be stretched, overlapped or interlocked. Shorter bivalents (M6, M7 and S8) complete pairing quite early and show full pairing in zygotene cells where about 30 per cent of the complement is asynapsed. When paired, these shorter bivalents display similar synaptonemal complex (SC) lengths (J. L. Santos et al., unpublished observations). For this reason $\mathrm{S} 8 \mathrm{BB}$ and $\mathrm{S} 8 \mathrm{BS}$ individuals, chosen for the analysis of pairing progression, were homozygotes, with or without C-heterochromatin, for M6 and M7 chromosomes.

To estimate the position of unpaired regions throughout M6-S8 bivalents in S8 BB and S8 BS individuals, paired and unpaired regions were

(a)

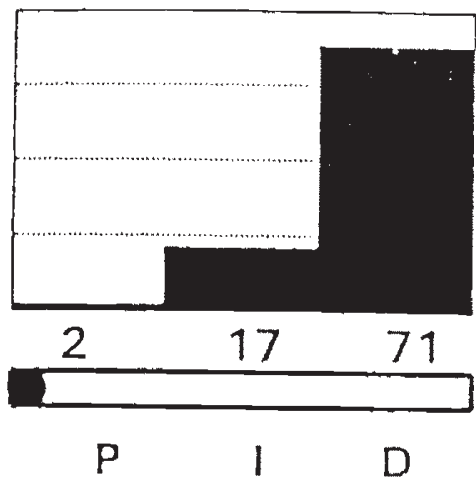

Chiasma location (b)

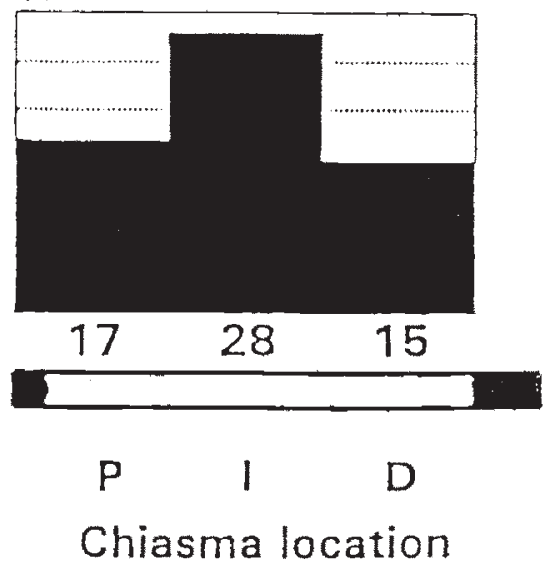

Fig. 2 Chiasma distributions in the S8 bivalent of Chorthippus jacobsi. (a) basic homozygotes (b) heterozygotes. 
measured in zygotene nuclei, expressed as percentages of bivalent lengths and plotted on the standard karyotype derived from pachytene SC measurements (J. L. Santos et al., unpublished observations). The results obtained are shown in Fig, 3. In S8 BB males, the ends of the short arms of M6-S8 bivalents were usually asynapsed and in some nuclei the centromeres too, indicating that pairing starts at or near the telomeric region of the long arms. However, two pairing initiation points, located at both ends of long arms, have also been observed. In S8 BS individuals one bivalent displaying unequal axis lengths was observed which represents the $\mathrm{S} 8$ bivalent heterozygous for the supernumerary segment. In this bivalent the pattern of pairing initiation changes completely because it starts at or near the short arm and proceeds towards the telomeric end of the long arm (Fig. $4 \mathrm{a}-\mathrm{c}$ ). In two nuclei the longer axial element showed a region of fold-back pairing (Fig. 4b). When pairing progresses the differences in length between axial elements decrease $(r=-0.87$, d.f. $=6)$ in such a way that at late zygotene $(70-80$ per cent pairing) M6-S8 bivalents exhibit fully paired synaptonemal complexes with equal axis lengths (Fig. 4d). This implies that in these bivalents a variable proportion of non-homologous pairing is achieved before pachytene where crossings-over occur.

\section{Euchorthippus pulvinatus}

The karyotype of this species is similar to that reported for Ch. jacobsi in terms of chromosome number and morphology but it shows a different C-banding pattern.
$M 6,57,58$ (a)

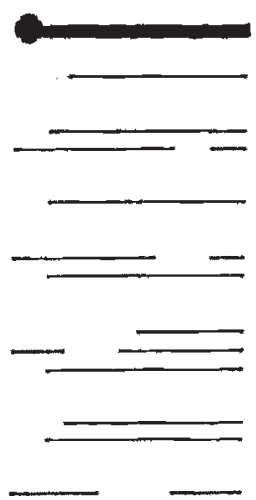

S8

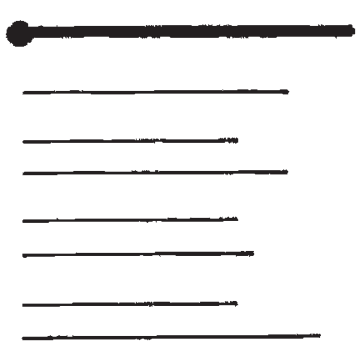

(b)
Fig. 3 The relative lengths and positions of paired regions (thin lines) in M6-S8 bivalents of $C h$. jacobsi from mid-late zygotene nuclei plotted on the pachytene SC karyotype. (a) Basic homozygotes for S8 chromosome (BB). Data taken from J. L. Santos et al. (unpublished results). (b) Segment heterozygotes for S8 chromosome (BS).
Thus, the M7 chromosome can have a thick C-band near the telomere in the Valdemoro population (Santos \& Giráldez, 1982) and the S8 chromosome can have a thin interstitial C-band near the centromere.

M7 and S8 bivalents are invariably monochiasmate. The chiasma distributions in two classes of M7 bivalents (BB and $\mathrm{BS}$ ) are shown in Fig. 5. In M7 BS bivalents chiasmata tend to be located close to the centromeric regions (Fig. 6a), although a relatively high frequency of distal associations, which are of chiasmate nature (Santos \& Esteban, 1990), have also been observed (Fig. 6b). In contrast, in M7 BB bivalents chiasmata are located all along the chromosome.

Analysis of silver-stained SCs in M7 BB bivalents indicated that synapsis at zygotene is either unidirectional from the telomeres of the long arms towards the centromeric regions and the short arms or bidirectional from initiation at or near both telomeres. Among the shorter bivalents, M7 can be identified because there are considerable differences in length with respect to M6. On the other hand, the S8 bivalent carries a nucleolar organizer region.

Only seven nuclei of the M7 BS male could be analysed: three were at late zygotene with 79,86 and 90 pairing percentages respectively and the other four were at pachytene with haploid SC lengths of 972,957 , 902 and $885 \mu \mathrm{m}$. In two of the zygotene nuclei (79 and 90 per cent paired) and in one of pachytene $(957 \mu \mathrm{m})$, both ends of M7 bivalent were synapsed resulting in a buckle comprising axes of unequal length. The size of the buckle was 14 and 11 per cent of the longer axis length in the zygotene nuclei (Fig. 7a-b) and 4 per cent in the pachytene nucleus (Fig. $7 \mathrm{c}-\mathrm{d}$ ). Then, it is apparent that the size of the loop decreases from zygotene to pachytene. Its distance to the nearest telomere is 23,18 and 4 per cent of the longer axis length respectively. In the remaining nuclei straight pairing can be observed (Fig. 7e). We conclude that, as in $C h$. jacobsi, certain chromosome regions of this bivalent show non-homologous pairing before or at the presumed time of chiasma formation.

No surface spread zygotene nuclei from the two individuals heterozygous for the S8 chromosome were obtained. All pachytene nuclei analysed (10) revealed straight-paired synaptonemal complexes with equal lengths. Therefore, heterosynapsis for the interstitial C-band is achieved at or before this stage.

\section{Discussion}

The electron microscopic analysis of SCs revealed that in the grasshopper species, analysed here, the heterozygosity for supernumerary segments changes the normal pattern of synapsis within the bivalents carrying 

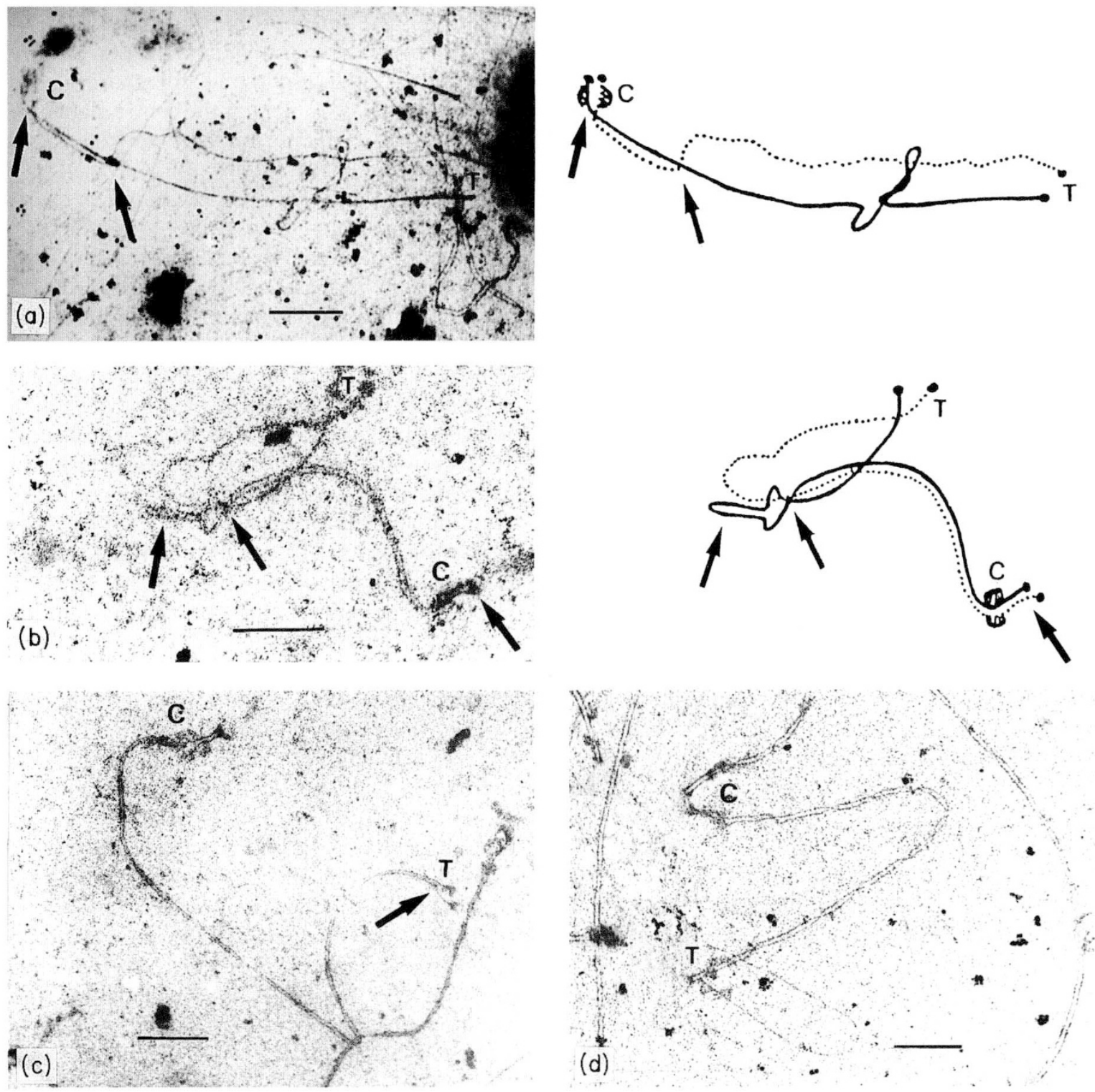

Fig. 4 Electron micrographs of silver stained SC preparations of Ch. jacobsi showing patterns of synapsis in the heteromorphic S8 bivalent. (a) Zygotene SC exhibiting unequal axis lengths; the paired region (between arrows) corresponds to that near the centromere. (b) Zygotene SC with fold-back pairing in the longer axis. Regions showing homologous and non-homologous synapsis are arrowed. (c) Zygotene SC with asynapsed portions of the pairing axes of unequal length (arrowed). (d) Pachytene SC showing full pairing and equalized axes. $\mathrm{C}=$ centromere, $\mathrm{T}=$ telomere. Bars represent $2.5 \mu \mathrm{m}$.

them. In S8 BB bivalents of Chorthippus jacobsi males, synapsis usually starts at or near the distal ends of long arms; however, in S8 BS bivalents synapsis is initiated at or near the distal ends of short arms and proceeds to the ends of the long arms in an unidirectional fashion followed by heterosynapsis involving the additional C-heterochromatic segment (Fig. 3). It is feasible that, as has been observed in Chloealtis conspersa (Moens et al., 1989), misalignment of heteromorphic cores and subsequent synaptic failure as a consequence of their attachment to the nuclear envelope can be involved in this process.

At late zygotene S8 BS bivalents show equalized axes. Therefore, a certain degree of non-homologous pairing in the proximity of the extra segment takes place before pachytene. Cross-over formation between 
(a)
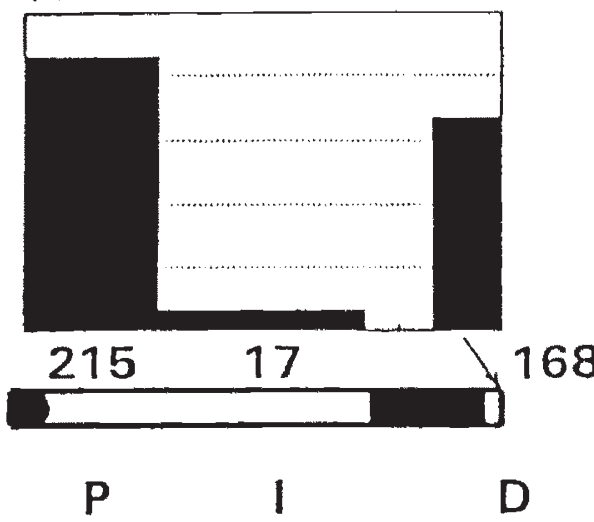

Chiasma location (b)

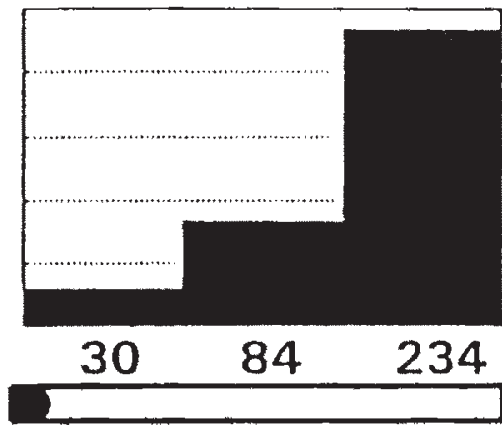

P $\quad 1$

Chiasma location

Fig. 5 Chiasma distributions in the M7 bivalent of Euchorthippus pulvinatus. (a) Basic homozygotes. (b) Heterozygotes. Data taken from J. L. Santos et al. (unpublished results).
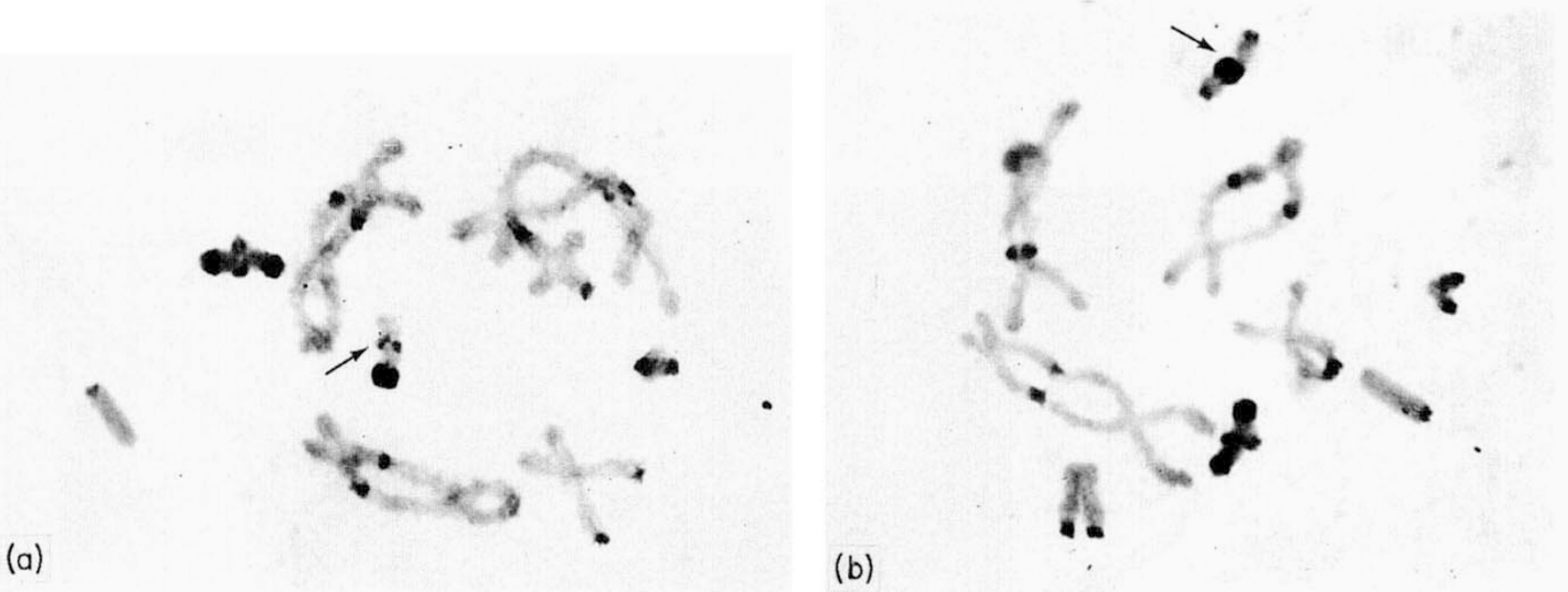

Fig. 6 C-banded diplotenes from a E. pulvinatus male heterozygous for a supernumerary segment in M7 bivalent. (a) M7 bivalent showing a proximal chiasma (arrowed). (b) M7 bivalent showing a distal chiasma (arrowed).

strictly homologous regions, mostly proximal and interstitial ones, would lead to the chiasma distribution displayed by these bivalents (Fig. 2).

Comparable situations involving an accommodation mechanism, which functions to equalized the lengths of the two axes of the heterochromatic synaptonemal complexes, have been reported in deer mice heterozygous for the presence of heterochromatic short arms (Hale \& Greenbaum, 1988a,b). In contrast, no evidence for an adjustment effect reducing the telomere length differences throughout pachytene-diplotene was found in rye bivalents heterozygous for telomeric C-bands. Furthermore, heterozygosity for the $1 \mathrm{RL}$ terminal $\mathrm{C}$-band did not modify the chiasma distribu- tion pattern of this arm (Gillies \& Lukaszewski, 1989). The large number of pairing initiation sites in plants compared to that displayed by the short chromosomes of rodents and grasshoppers may provide an explanation for these contradictory results.

Light microscope observations in plants of Scilla siberica, heterozygous for interstitial C-bands, indicated that heterochromatin interferes with euchromatic pairing at later stages of pachytene leading to the formation of a loop produced by the non-pairing adjacent euchromatic segment. Berger \& Greilhuber (1991) concluded that this segment would be excluded from recombination. On the other hand, SC observations in plants of rye heterozygous for an interstitial C-band on 


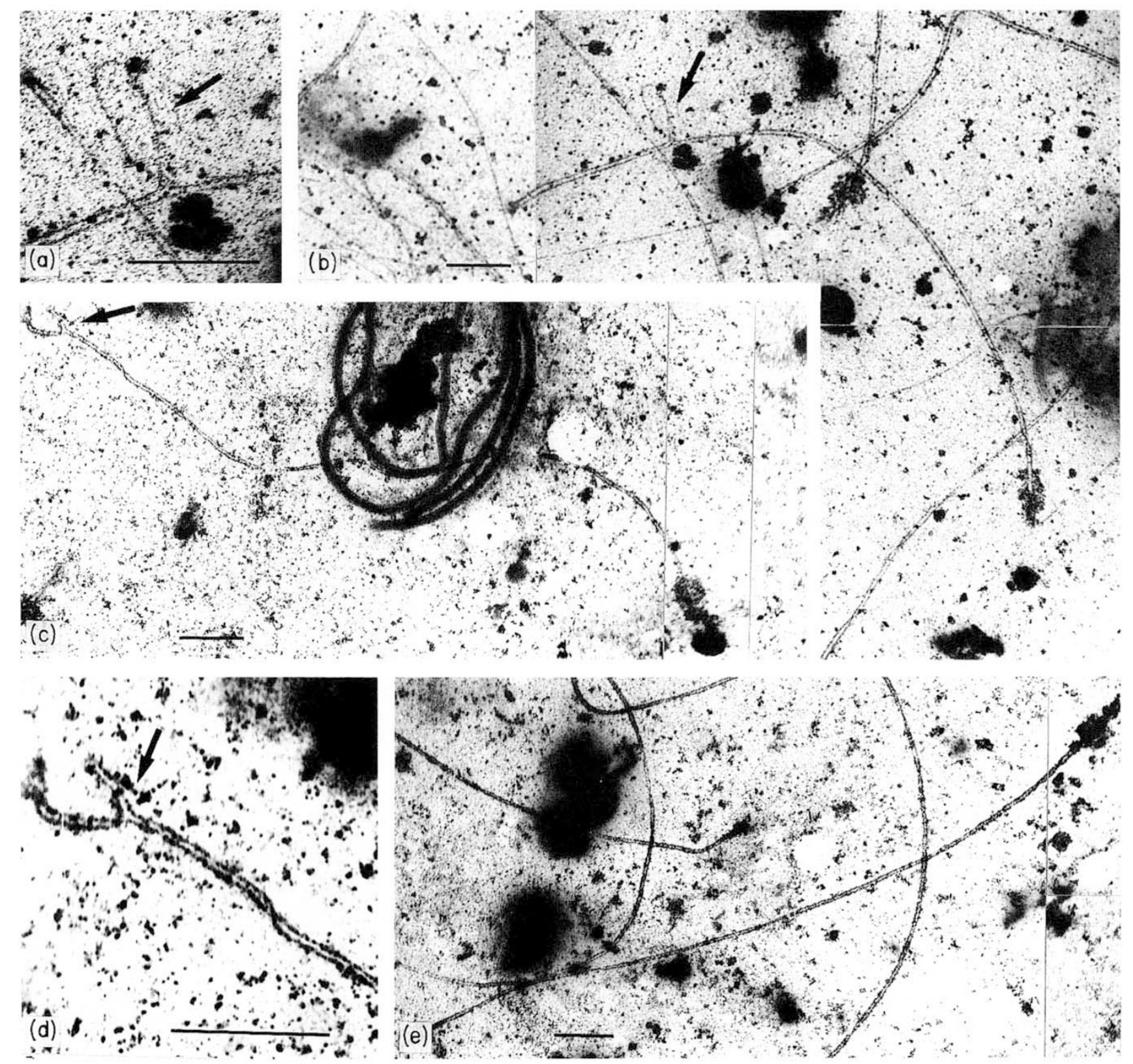

Fig. 7 Electron micrographs of silver stained SC preparations of E. pulvinatus showing patterns of synapsis in the heteromorphic M7 bivalent. (a) Detail of the buckle in (b). (b) Zygotene bivalent showing a buckle configuration (arrowed), both ends are synapsed. (c) Detail of the buckle in (d). (d) Pachytene bivalent showing a partially adjusted buckle (arrowed). (e) Pachytene bivalent showing axes with equal length and absence of buckle. Bars represents $2.5 \mu \mathrm{m}$.

chromosome 6R indicated the existence of heterosynapsis following alignment of the $\mathrm{C}$-band region and these heteromorphic bivalents showed a chiasma redistribution when compared with non C-band carriers (L. E. Benavente, unpublished results).

Similar results have been reported in animals, thus heterosynapsis at an interstitial C-band heterozygosity took place in the red kangaroo Macropus rufus (Sharp, 1986), in the rodent Peromyscus beatae (Sudman et al.,
1989) and in the grasshopper Euchorthippus pulvinatus (this work). Whereas the dynamics of the process is unknown in the S8 bivalent of the latter species, it is clear that in the M7 bivalent synapsis is bidirectional from both telomeres leading to a buckle formation. If we consider the size and the position of the buckle (Fig. 7b and d) it appears that it can be formed in different locations in the bivalent. This conclusion is based on the observation that the maximum 
distance observed from the buckle to the nearest telomere is higher than the maximum size loop. On the contrary, if the buckle was always formed at the same position and subsequently readjusted, an increase in the distance from the buckle to the telomere would be expected during this process but this has not been observed. Therefore, a certain extent of pairing around the buckle must be non-homologous. This hypothesis is reinforced when the euchromatin-heterochromatin ratio in M7 mitotic chromosomes is considered (about 1:1), although heterochromatin may be underrepresented in the SC (Stack, 1984). Nevertheless, by pachytene and in some nuclei by late zygotene the lateral elements of the heteromorphic bivalent display the same length. As chiasmata are formed between homologous chromosome regions and a variable proportion of non-homologous pairing involves the $\mathrm{C}$-band location and surroundings, one would expect a high frequency of proximal and distal chiasmata, as observed (Fig. 5).

In conclusion, the heterozygosity for interstitial and telomeric supernumerary segments produce different variations in the synaptic patterns of the carrier bivalents when compared with non-carriers. However, at late zygotene or early pachytene heterosynapsis is achieved in both cases which implies a certain degree of non-homologous pairing. The absence of chiasmata in regions near the segments, those in which nonhomologous pairing took place, would lead to the chiasma distributions observed in these heteromorphic bivalents. In this context, the dependence of the intensity of chiasma redistribution on the size of the extra segment found by Navas-Castillo et al. (1987), could be explained on the basis that small size segments produce less non-homologous pairing in bivalents carrying them than large ones.

\section{Acknowledgements}

This paper was supported by a grant PB 88-0121 awarded by Comision Interministerial de Ciencia y Tecnologia (Spain). Dr Nieves Cuñado, José Barrios, Agustin Fernández and the staff of the Complutense University Electron Microscope Unit, especially Dr Carlos Barba, provided valuable assistance.

\section{References}

BERGER, R. AND GREILHUBER, J. 1991. C-bands and chiasma distribution in Scilla siberica (Hyacinthaceae). Genome, 34, 179-189.
CROFT, J. A. AND JONES, G. H. 1986. Surface spreading of synaptonemal complexes in locusts. I. Pachytene observations. Chromosoma, 93, 483-488.

GILLIES, C. B. AND LUKASZEWSKI, A. J. 1989. Synaptonemal complex formation in rye (Secale cereale) heterozygous for telomeric C-bands. Genome, 32, 901-907.

hale, D. W. AND GReEnbaum, I. F. 1988a. Chromosomal pairing in deer mice heterozygous for the presence of heterochromatic short arms. Genome, 30, 44-47.

HALE, D. W. AND GREENBAUM, I. F. 1988b. Synapsis of a chromosomal pair heterozygous for a pericentric inversion and the presence of a heterozygous short arm. Cytogenet. Cell Genet., 48, 55-57.

HEWITT, G. M. AND JOHN, B. 1968. Parallel polymorphism for supernumerary segments in Chorthippus parallelus. I. British populations. Chromosoma, 25, 319-342.

JOHN, B. AND KING, M. 1985. The inter-relationship between heterochromatin distribution and chiasma distribution. Genetica, 66, 183-194.

MIKLOS, G. L. C. AND NANKIVELL, R. N. 1976. Telomeric satellite DNA functions in regulating recombination. Chromosoma, 56, 143-167.

MOENS, P. B., BERNELOT-MOENS, C. AND SPYROPOULOS, B. 1989. Chromosome core attachment to meiotic nuclear envelope regulates synapsis in Chloealtis (Orthoptera). Genome, 32, 601-610.

NAVAS-CASTILLO, J., CABRERO, J. AND CAMACHO, J. P. M. 1985. Chiasma redistribution in bivalents carrying supernumerary chromosome segments in grasshoppers. Heredity, 55, 245-248.

NAVAS-CASTILlo, J., CABRERO, J. AND CAMACHO, J. P. M. 1987. Chiasma redistribution in presence of supernumerary chromosome segments in grasshoppers: dependence on the size of the extra segment. Heredity, 58, 409-414.

RIVA, E., FOX, D. P., GIRALDEZ, R. AND SANTOS, J. L. 1984. Chiasma frequency and distribution in the presence and absence of supernumerary chromosome segments in the grasshopper Euchorthippus pulvinatus gallicus. Heredity, 53, 101-106.

SANTOS, J. L. AND ESTEBAN, M. R. 1990. On the nature of meiotic associations involving heterochromatic ends in grasshoppers. Genome, 33, 725-728.

SANTOS, J. L. AND GIRALDEZ, R. 1978. The effect of C-heterochromatin in chiasma terminalization in Chorthippus biguttulus L. (Acrididae, Orthoptera). Chromosoma, 70, 59-66.

SANTOS, J. L. AND GIRALDEZ, R. 1982. C-heterochromatin polymorphism and variation in chiasma localization in Euchorthippus pulvinatus (Acrididae, Orthoptera). Chromosoma, 85, 507-518.

SHARP, P. J. 1986. Synaptic adjustment at a C-band heterozygosity. Cytogenet. Cell Genet., 41, 56-57.

STACK, S. M. 1984. Heterochromatin, the synaptonemal complex and crossing-over. $J$. Cell Sci., 71, 159-176.

SUDMAN, P. D., GREENBAUM, I. F., HALE, D. W. AND SMITH, S. A. 1989. Synaptic adjustment in Peromyscus beatae (Rodentia: Cricetidae) heterozygous for interstitial heterochromatin. Cytogenet. Cell Genet., 50, 1-5. 